\title{
Molecular gas in compact galaxies
}

\author{
F. P. Israel
}

\author{
Sterrewacht Leiden, PO Box 9513, 2300 RA Leiden, The Netherlands \\ e-mail: israel@strw.leidenuniv.nl
}

Received 22 October 2004 / Accepted 21 April 2005

\begin{abstract}
New observations of eleven compact galaxies in the ${ }^{12} \mathrm{CO} J=2-1$ and $J=3-2$ transitions are presented. From these observations and literature data accurate line ratios in matched beams have been constructed, allowing the modelling of physical parameters. Matching a single gas component to observed line ratios tends to produce physically unrealistic results, and is often not possible at all. Much better results are obtained by modelling two distinct gas components. In most observed galaxies, the molecular gas is warm $\left(T_{\mathrm{k}}=50-150 \mathrm{~K}\right)$ and at least partially dense $\left(n\left(\mathrm{H}_{2}\right) \geq 3000 \mathrm{~cm}^{-3}\right)$. Most of the gas-phase carbon in these galaxies is in atomic form; only a small fraction $(\sim 5 \%)$ is in carbon monoxide. Beam-averaged CO column densities are low (of the order of $10^{16} \mathrm{~cm}^{-2}$ ). However, molecular hydrogen column densities are high (of the order of $10^{22} \mathrm{~cm}^{-2}$ ) confirming large CO-to- $\mathrm{H}_{2}$ conversion factors (typically $X=10^{21}-10^{22} \mathrm{~cm}^{-2} / \mathrm{K} \mathrm{km} \mathrm{s}^{-1}$ ) found for low-metallicity environments by other methods. From CO spectroscopy, three different types of molecular environment may be distinguished in compact galaxies. Type I (high rotational and isotopic ratios) corresponds to hot and dense molecular clouds dominated by star-forming regions. Type II has lower ratios, similar to the mean found for infrared-luminous galaxies in general, and corresponds to environments engaged in, but not dominated by, star-forming activity. Type III, characterized by low ${ }^{12} \mathrm{CO}(2-1) /(1-0)$ ratios, corresponds to mostly inactive environments of relatively low density.
\end{abstract}

Key words. galaxies: abundances - ISM: abundances - ISM: molecules - galaxies: irregular - submillimeter

\section{Introduction}

Compared to large spiral galaxies, dwarf and compact galaxies are difficult to detect in molecular lines, even in those of the relatively abundant $\mathrm{CO}$ molecule. However, molecular clouds constitute the unique environment out of which the stars are formed and knowledge of their occurrence, spatial distribution, mass and physical condition is essential to gain insight in the process of star formation in galaxies. For this reason, many surveys of dwarf and compact galaxies have been conducted over the last decades, although generally with low detection rates (Table 1).

Detection rates are highest for "tidal" dwarfs (cf. Braine et al. 2001), "big" dwarfs (Sm) and galaxies that are only modestly metal-poor. They are lowest for "small" dwarfs (Im), blue compact dwarf galaxies (BCDGs) and practically non-existent for very metal-poor dwarfs (Taylor et al. 1998; Barone et al. 2000). In the extensive survey by Albrecht et al. (2004), detection rates are $72 \%$ and $39 \%$ for Sm and Im galaxies respectively (see also Leroy et al. 2005).

Velocity-integrated ${ }^{12} \mathrm{CO}(1-0)$ fluxes are often combined with the so-called $\mathrm{CO}-$ to- $\mathrm{H}_{2}$ conversion factor $X$ to derive a beam-averaged $\mathrm{H}_{2}$ column-density, hence an estimate for the $\mathrm{H}_{2}$ mass in the beam. It is widely (but not yet universally) accepted that this factor $X$ is a function of environmental conditions (such as metallicity, radiation density etc.). However, the extent to which $X$ is sensitive to e.g. changes in metallicity is still a matter of debate. Arguments for a strong dependence of $X$ on metallicity as traced by the oxygen abundance $[\mathrm{O}] /[\mathrm{H}]$ have been presented by Israel $(1997,2000)$, Barone et al. (2000) and Boselli et al. (2002).

Obviously, it is much preferable to determine actual molecular column densities and masses from an analysis of the physical condition of the gas than from an empirically determined factor that relates column density to the flux of an optically thick line. In principle, multi-line observations can be used as a diagnostic tool to estimate the physical condition of the gas, but this requires that the various transitions have been observed at the same celestial position with matched beamsizes. A glance at Table 1 shows that only a few surveys sample CO line transitions other than $J=1-0$. In a number of surveys, $J=2-1$ and $J=1-0$ measurements were made simultaneously with the same telescope, therefore with beam areas differing by a factor of four. Mapping the $J=2-1$ line over the extent of the $J=1-0$ beam would make it possible to compare the two transitions, but this is rarely done. Only the very recent survey by Albrecht et al. combines measurements in the two transitions obtained with the IRAM $30 \mathrm{~m}$ and the SEST $15 \mathrm{~m}$ apertures, providing matched (SEST) $J=2-1$ and (IRAM) $J=1-0$ measurements. In all preceding surveys, including the $\mathrm{CSO}{ }^{12} \mathrm{CO}(3-2)$ survey by Meier et al. (2001), line ratios could only be estimated as a function of (assumed) source 
Table 1. CO surveys of dwarf and compact galaxies.

\begin{tabular}{lrrr}
\hline \hline Reference & \multicolumn{2}{c}{ Number } & Line \\
& Obs. & Det. & \\
\hline Elmegreen et al. (1980) & 7 & 1 & $1-0$ \\
Gordon et al. (1982) & 7 & 0 & $1-0$ \\
Israel \& Burton (1986) & 12 & 0 & $1-0$ \\
Thronson \& Bally (1987) & 22 & 10 & $1-0$ \\
Tacconi \& Young (1987) & 15 & 6 & $1-0$ \\
Arnault et al. (1988) & 12 & 0 & $1-0$ \\
Sage et al. (1992) & 15 & 8 & $1-0 / 2-1$ \\
Hunter \& Sage (1993) & 5 & 0 & $1-0 / 2-1$ \\
Israel et al. (1995) & 25 & 6 & $1-0$ \\
Gondhalekar et al. (1998) & 29 & 5 & $1-0$ \\
Taylor et al. (1998) & 11 & 3 & $1-0$ \\
Barone et al. (2000) & 10 & 2 & $1-0 / 2-1$ \\
Meier et al. (2001) & 8 & 8 & $3-2$ \\
Braine et al. (2001) & 10 & 8 & $1-0 / 2-1$ \\
Boselli et al. (2002) & 6 & 1 & $1-0$ \\
Albrecht et al. (2004) & 64 & 41 & $1-0 / 2-1$ \\
Leroy et al. (2005) & 121 & 47 & $1-0$ \\
\hline
\end{tabular}

extent (point source, extended source, or inbetween). As can easily be seen, this introduces very large uncertainties, effectively ruling out meaningful modelling of physical parameters.

In this paper we present new observations, which together with measurements published in the literature, allow us for the first time to construct reliable line ratios in matched beams for a sample of about a dozen dwarf or compact galaxies.

\section{Observations}

All observations described in this paper were made with the $15 \mathrm{~m}$ James Clerk Maxwell Telescope (JCMT) on Mauna Kea (Hawaii) ${ }^{1}$, mostly between July 1995 and December 1996. They were made in beamswitching mode with a throw of $3^{\prime}$ in azimuth using the DAS digital autocorrelator system. When sufficient free baseline was available (i.e. when the detected line was sufficiently narrow), we subtracted second or third order baselines from the profiles. In all other cases, linear baseline corrections were applied. All spectra were scaled to a main-beam brightness temperature, $T_{\mathrm{mb}}=T_{\mathrm{A}}^{*} / \eta_{\mathrm{mb}}$. We used values of $\eta_{\mathrm{mb}}$ appropriate to the epoch of observation. These values were between 0.64 and 0.72 at $230 \mathrm{GHz}$, between 0.53 and 0.60 at $345 \mathrm{GHz}$, and between 0.50 and 0.53 at $461 \mathrm{GHz}$. Spectra of observed positions are shown in Fig. 1. We also made small maps of the four galaxies (IC 10,

\footnotetext{
1 The James Clerk Maxwell Telescope is operated on a joint basis between the United Kingdom Particle Physics and Astrophysics Council (PPARC), the Netherlands Organisation for Scientific Research (NWO) and the National Research Council of Canada (NRC).
}

Haro 2, NGC 4194 and NGC 6052) distinguished by relatively strong $J=3-2{ }^{12} \mathrm{CO}$ line emission. These maps are shown in Figs. 2 and 3.

\section{Results}

\subsection{Detections}

Details of the observed galaxies and the observational results obtained are summarized in Table 2. In this table, Col. 1 gives the names, with alternative names in the notes at bottom. Right ascensions and declinations in Cols. 2 and 3 are those of the observed $(0,0)$ position. The receivers were tuned to the radial velocity given in Col. 4; as is obvious from Fig. 1 this was usually very close to the $\mathrm{CO}$ velocity. Column 6 gives the integrated absolute blue magnitude as retrieved from photometric data in the NASA-IPAC NED and calculated for the assumed galaxy distance listed in Col. 5. there. Columns 7 and 8 identify the observed ${ }^{12} \mathrm{CO}$ transition and the concomitant FWHM beamsize. Finally, Cols. 9 and 10 present the observed peak main-beam brightness temperature, and the CO intensity integrated over the observed profile. Quoted errrors are rms values; upper limits are three times the rms value.

As Fig. 1 and Table 2 show, emission in the $J=$ $2-1{ }^{12} \mathrm{CO}$ transition was detected from all observed galaxies, although the emission from $2 \mathrm{Zw} 40$ was close to the detection threshold. $2 \mathrm{Zw} 40$ was not detected in the $J=3-2$ transition nor was NGC 2537 in spite of its clear detection in the $J=2-1$ transition. By convolving the maps of IC 10, Haro 2, NGC 4194 and NGC 6052 to a resolution of $21^{\prime \prime}$, we also obtained for these galaxies the $J=3-2{ }^{12} \mathrm{CO}$ intensity matched to the $J=2-1$ beamsize (Table 2 ).

\subsection{Line ratios}

We have determined rotational line ratios by directly comparing transitions observed with beams of identical or very similar size, using our own measurements and results from the literature, summarized in Table 3. Where observations in identical beams were lacking, we extrapolated $\mathrm{CO}$ fluxes to matching beamsizes. This was done either directly by plotting observed CO flux (Tables 2 and 3) as a function of beamsize and reading off the desired flux value at the relevant beamsize, or indirectly by using the equivalent $\mathrm{CO}$ source size. The equivalent size can be determined from observations with different beams (and not necessarily in the same transition) under the assumption that the $\mathrm{CO}$ emission has a radial Gaussian brightness distribution with circular symmetry. It can also be estimated from high-resolution (array) maps of the $\mathrm{CO}$ distribution. Actual methods used are found in Sect. 3.3 dealing with the individual galaxies and the resulting ratios are given in Table 4, together with metallicities $[\mathrm{O}] /[\mathrm{H}]$ taken from data compiled by Taylor et al. (1988) and Meier et al. (2001) and the IRAS far-infrared $60 \mu \mathrm{m} / 100 \mu \mathrm{m}$ flux-density ratios taken from Lonsdale et al. (1989). Table 4 consists of three parts: the top section contains the galaxies observed by us, whereas the remainder consists of data culled from the literature. The bottom section contains galaxies experiencing starburst activity. 

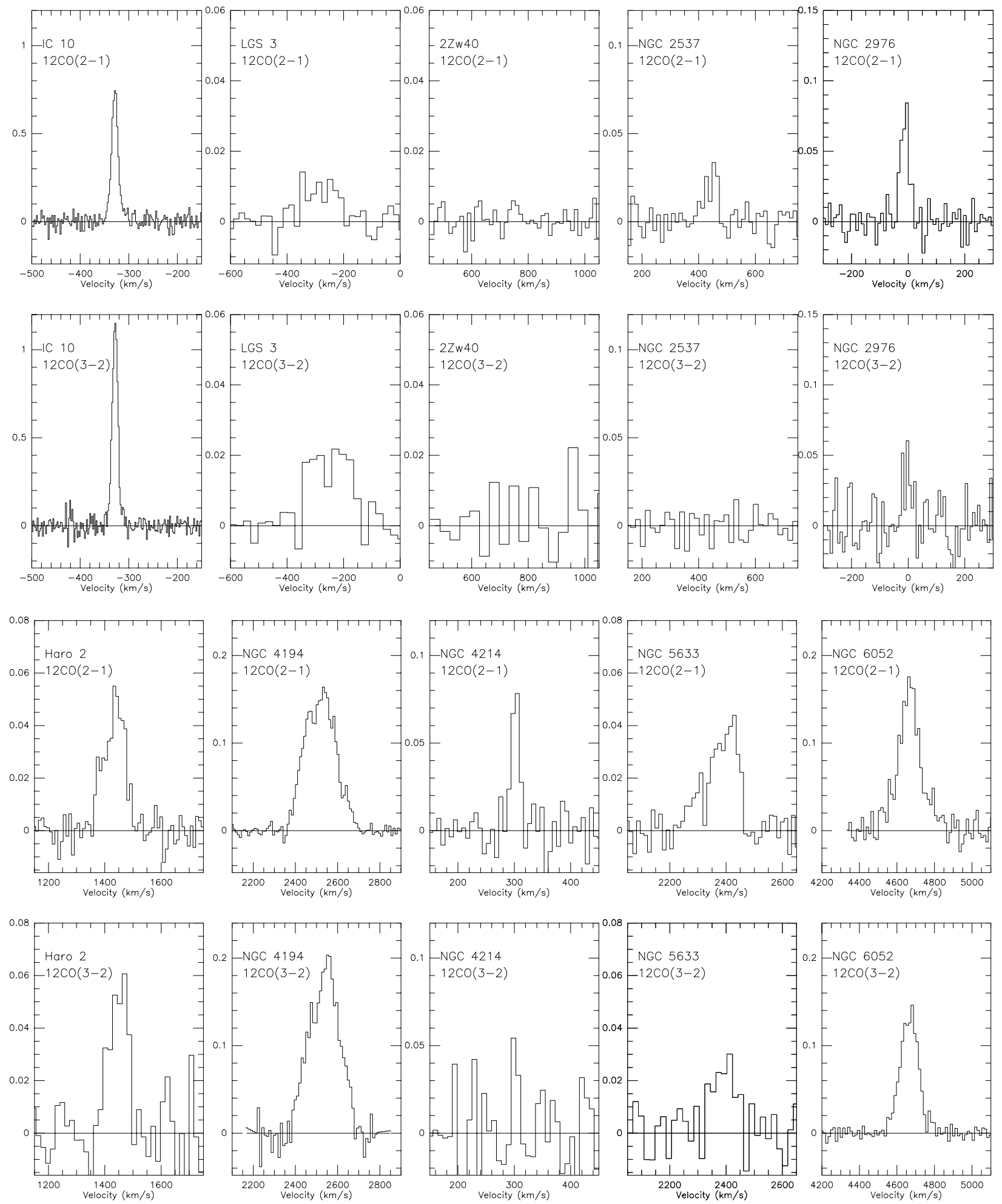

Fig. 1. $J=2-1$ and $J=3-2{ }^{12} \mathrm{CO}$ emission-line spectra observed towards the sample dwarf and peculiar galaxies. Horizontal scale is $V_{\mathrm{LSR}}$ in $\mathrm{km} \mathrm{s}^{-1}$, vertical scale is main-beam brightness temperature $T_{\mathrm{mb}}$ in Kelvins.

Not included in Table 4 is the dwarf galaxy UM 465 for which CO measurements imply a $(2-1) /(1-0)$ ratio of $1.16 \pm$ 0.29 (Sage et al. 1992; Barone et al. 2000) and recently published results by Albrecht et al. (2004) which allow direct determination of the 2-1/1-0 ratio for another six dwarf galaxies: NGC 145 (0.74 \pm 0.13$)$; NGC $2730(0.62 \pm 0.17)$;
NGC $4532(0.59 \pm 0.12)$; NGC $6570(0.41 \pm 0.12)$; NGC 7732 $(0.49 \pm 0.24)$; IC $3521(0.49 \pm 0.14)$ as well as upper limits for four more: NGC $178(\leq 0.77)$; NGC $1140(\leq 1.36)$; NGC 3659 $(\leq 0.17)$; NGC $4234(\leq 0.80)$.

On average, $\mathrm{CO}$ emission from compact galaxies is weak. As emission from the ${ }^{13} \mathrm{CO}$ isotope is usually at least an order 

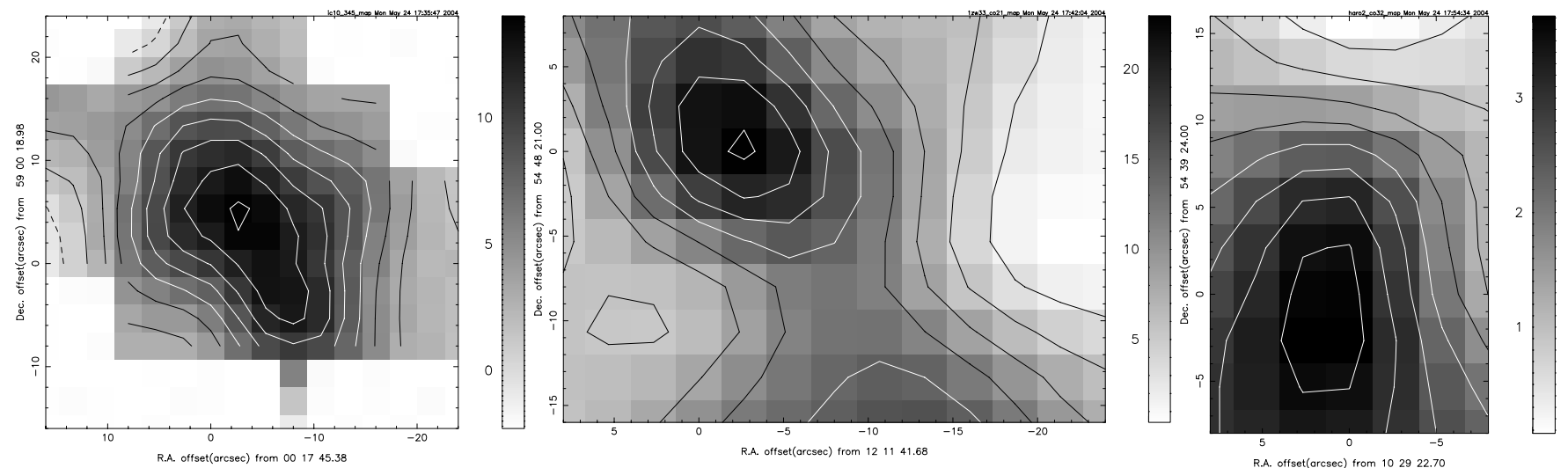

Fig. 2. Contour maps of integrated CO emission. Lowest contour is always at zero. Left: $\mathrm{IC} 10(J=3-2)$ with main-beam brightness temperature contours in multiples of $1 \mathrm{~K}$. Center: NGC $4194(J=2-1)$ with contours in multiples of $4 \mathrm{~K}$. Right: Haro $2(J=3-2)$ with contours in multiples of $0.75 \mathrm{~K}$.

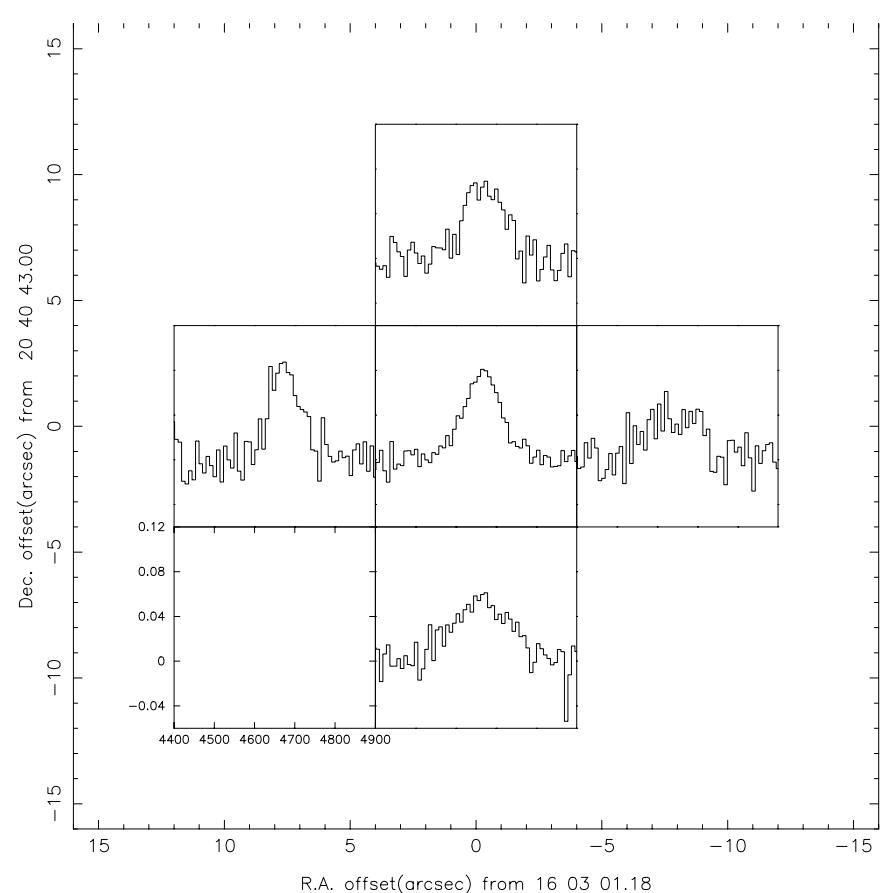

Fig. 3. Grid map of the $J=3-2$ CO emission from NGC 6052. Horizontal scale is radial velocity $V_{\mathrm{LSR}}$ in $\mathrm{km} \mathrm{s}^{-1}$, vertical scale is main-bean brightness temperature $T_{\mathrm{mb}}$ in Kelvins.

of magnitude less than that from ${ }^{12} \mathrm{CO}$, very few galaxies have been measured in this line. In Table 5 we list the available information on the galaxies from Table 4 . In effect, this sample is limited to actively star-forming galaxies.

\subsection{Individual galaxies}

NGC 55 is a small SB(s)m galaxy, roughly as we would expect to see the LMC edge-on, member of the nearby Sculptor Group and rich in interstellar gas. Dettmar \& Heithausen (1989) used the SEST to make a small $J=1-0{ }^{12} \mathrm{CO}$ map of the brightest part. At the position of the $\mathrm{CO}$ peak in this map, Becker \& Freundling (1991) also measured the $J=1-0{ }^{13} \mathrm{CO}$ intensity. No further $\mathrm{CO}$ data on this interesting galaxy have appeared in the literature. Our $J=2-1$ spectrum (not shown) was taken very close to the $\mathrm{CO}$ peak, at the same position as our earlier $J=1-0$ spectrum (Israel et al. 1995).

IC 10 (dIrr IV) is an unusual Local Group galaxy; it is one of the most luminous dwarf galaxies in the far-infrared (Melisse \& Israel 1994), and appears to be the nearest representative of the class commonly referred to as Blue Compact Galaxies (BCG). It contains two major groupings of HII regions (see Hodge \& Lee 1990; Yang \& Skillman 1993; Chyzy et al. 2003), the brightest of which is alternatingly known in the literature as IC $10 \mathrm{~A}$ or IC 10-SE. The map in Fig. 2 shows the CO distribution associated with IC 10-SE, which resembles that of far-infrared and submillimeter emission from heated dust (Thronson et al. 1990) and that of ionized carbon ([CII]) emission (Madden et al. 1997). Studies of the dense molecular medium in IC 10-SE were published by Petitpas \& Wilson (1998) and Bolatto et al. (2000). The $(2-1) /(1-0)$ ratio in Table 4 was taken from the former; the $(3-2) /(2-1)$ ratio is the mean of their determination and our higher value.

NGC 1569 (IBm, Sbrst) is an extreme starburst dwarf galaxy (Israel 1988). Weak CO detected by various authors (Greve et al. 1996; Taylor et al. 1998, 1999; Meier et al. 2001; Albrecht et al. 2004) has been mapped and discussed in Mühle's $2003 \mathrm{Ph} . \mathrm{D}$. thesis from which we took the ratios in Table 4 (average of values from her Table 3.9). The extent of $\mathrm{CO}$ emission is slightly less than $30^{\prime \prime}$.

LGS 3 (dIrr/dSph), a Local Group galaxy in Pisces, is one of the faintest dwarf galaxies known. It was surprisingly detected in CO by Tacconi \& Young (1987) with a $45^{\prime \prime}$ beam. Our measurements were made at the same position, but with smaller beams. The range of ratios in Table 4 was obtained by assuming the $\mathrm{CO}$ source to be pointlike or extended respectively.

$2 \mathbf{Z w} 40$ is a gas-rich galaxy alternately classified as a BCG or an HII merger galaxy (Beck et al. 2002, and references therein). CO was detected by Thronson \& Bally (1987), Tacconi \& Young (1987), and Sage et al. (1992). Our spectra were taken at the position used by the latter. The integrated intensities as a function of beamsize suggest an equivalent $\mathrm{CO}$ size of $23^{\prime \prime}$ which was used to obtain the $(2-1) /(1-0)$ ratio in Table 4. 
Table 2. JCMT ${ }^{12} \mathrm{CO}$ observations of compact galaxies.

\begin{tabular}{|c|c|c|c|c|c|c|c|c|c|}
\hline Galaxy & $\begin{array}{r}\text { RA(1950) } \\
\text { (h:m:s.s) }\end{array}$ & $\begin{array}{r}\operatorname{Dec}(1950) \\
(\mathrm{d}: \mathrm{m}: \mathrm{s})\end{array}$ & $\begin{array}{c}V_{\mathrm{LSR}} \\
\left(\mathrm{km} \mathrm{s}^{-1}\right)\end{array}$ & $\begin{array}{c}\text { Distance } \\
(\mathrm{Mpc})\end{array}$ & $\begin{array}{c}M_{B T}^{\circ} \\
(\mathrm{mag})\end{array}$ & Line & $\begin{array}{c}\text { Beam } \\
\left({ }^{\prime \prime}\right)\end{array}$ & $\begin{array}{r}T_{\mathrm{mb}} \\
(\mathrm{mK})\end{array}$ & $\begin{array}{c}I_{\mathrm{CO}} \\
\left(\mathrm{K} \mathrm{km} \mathrm{s}^{-1}\right)\end{array}$ \\
\hline NGC 55 & $00: 12: 30.5$ & $-39: 28: 55$ & +135 & 2.0 & -18.9 & $J=2-1$ & 21 & 75 & $2.5 \pm 0.2$ \\
\hline \multirow[t]{4}{*}{ IC $10-\mathrm{SE}$} & $00: 17: 45.4$ & $+59: 00: 19$ & -330 & 0.8 & -16.3 & $J=2-1$ & 21 & 735 & $13.0 \pm 0.3$ \\
\hline & & & & & & $J=3-2$ & 14 & 1130 & $17.5 \pm 0.2$ \\
\hline & & & & & & & 21 & 895 & $14.9 \pm 0.3$ \\
\hline & & & & & & $J=4-3$ & 11 & 490 & $9.4 \pm 0.9$ \\
\hline \multirow[t]{2}{*}{ LGS 3} & 01:01:12.0 & $+21: 37: 00$ & -300 & 0.7 & -10.0 & $J=2-1$ & 21 & 11 & $1.4 \pm 0.3$ \\
\hline & & & & & & $J=3-2$ & 14 & 28 & $3.5 \pm 0.6$ \\
\hline \multirow[t]{2}{*}{$2 \mathrm{Zw} 40$} & 05:53:05.0 & $+03: 23: 07$ & +770 & 10 & $-17:$ & $J=2-1$ & 21 & 6 & $0.4 \pm 0.15$ \\
\hline & & & & & & $J=3-2$ & 14 & $<16$ & $<0.8$ \\
\hline \multirow[t]{2}{*}{ NGC $2537^{a}$} & 08:09:42.8 & $+46: 08: 33$ & +440 & 6.9 & -17.2 & $J=2-1$ & 21 & 23 & $1.1 \pm 0.2$ \\
\hline & & & & & & $J=3-2$ & 14 & $<11$ & $<0.8$ \\
\hline \multirow[t]{2}{*}{ NGC 2976} & 09:43:10.0 & $+68: 08: 43$ & +5 & 3.5 & -17.2 & $J=2-1$ & 21 & 103 & $4.1 \pm 0.2$ \\
\hline & & & & & & $J=3-2$ & 14 & 48 & $1.4 \pm 0.4$ \\
\hline \multirow[t]{3}{*}{ Haro $2^{b}$} & $10: 29: 22.7$ & $+54: 39: 24$ & +1445 & 20 & -18.2 & $J=2-1$ & 21 & 52 & $4.5 \pm 0.2$ \\
\hline & & & & & & $J=3-2$ & 14 & 64 & $6.7 \pm 0.6$ \\
\hline & & & & & & & 21 & 52 & $3.4 \pm 0.5$ \\
\hline \multirow[t]{3}{*}{ NGC $4194^{c}$} & $12: 11: 41.7$ & $+54: 48: 21$ & +2506 & 39 & -20.1 & $J=2-1$ & 21 & 167 & $34.3 \pm 0.7$ \\
\hline & & & & & & $J=3-2$ & 14 & 213 & $39.3 \pm 2.5$ \\
\hline & & & & & & & 21 & 177 & $26.7 \pm 2.6$ \\
\hline \multirow[t]{2}{*}{ NGC 4214} & $12: 13: 11.2$ & $+36: 35: 47$ & +280 & 4.1 & -17.9 & $J=2-1$ & 21 & 86 & $1.7 \pm 0.2$ \\
\hline & & & & & & $J=3-2$ & 14 & 78 & $1.2 \pm 0.45$ \\
\hline \multirow[t]{2}{*}{ NGC $5633^{d}$} & $14: 25: 37.2$ & $+46: 22: 33$ & +2320 & 32 & -19.9 & $J=2-1$ & 21 & 36 & $5.2 \pm 0.7$ \\
\hline & & & & & & $J=3-2$ & 14 & 28 & $3.2 \pm 0.5$ \\
\hline \multirow[t]{3}{*}{ NGC $6052^{e}$} & 16:03:01.2 & $+20: 40: 39$ & +4725 & 65 & -20.7 & $J=2-1$ & 21 & 107 & $17.3 \pm 0.3$ \\
\hline & & & & & & $J=3-2$ & 14 & 151 & $18.0 \pm 0.8$ \\
\hline & & & & & & & 21 & 117 & $12.9 \pm 0.8$ \\
\hline
\end{tabular}

Note: ${ }^{a}$ Mkn $86=\operatorname{Arp~6;~}{ }^{b}$ Mkn $33=\operatorname{Arp~233;~}{ }^{c}$ Mkn $201=1$ Zw $33=\operatorname{Arp~160;~}{ }^{d} 1$ Zw 89; ${ }^{e}$ Mkn $297=\operatorname{Arp} 209$.

He 2-10 (I0pec, Sbrst) is a dwarf galaxy experiencing a strong starburst caused by an ongoing merger. The equivalent CO size is about $12^{\prime \prime}$, consistent with the OVRO $J=$ 1-0 CO map published by Kobulnicky et al. (1995). The $\mathrm{CO}$ ratios in Table 4 were taken from data in that paper, from Meier et al. (2001) and from Baas et al. (1994).

NGC 2537 ( $\mathrm{SB}(\mathrm{s}) \mathrm{m}$ pec) is also considered to be a BCG and often referred to as Mkn 86 or, after its optical appearance, as the Bear Claw. Not detected by Thronson \& Bally (1987), ${ }^{12} \mathrm{CO}$ emission was succesfully measured in the $J=1-0$ and $J=2-1$ transitions by Sage et al. (1992). Our measurements were made at the same position, and the line ratios follow directly from the data listed in Tables 2 and 3. NGC 2537 has also been mapped in $J=1-0$ and $J=2-1{ }^{12} \mathrm{CO}$ by Gil de Paz et al. (2002). Our ${ }^{12} \mathrm{CO}(2-1) /(1-0)$ ratio agrees with their less certain value $1.06 \pm 0.40$.

NGC 2976 (SAc pec, HII) is part of the M 81 group. Optically, it is similar to NGC 2537 and NGC 1569 in having a complex central structure surrounded by an extended and relatively featureless distribution of stars. Bright HII regions occur at either end of the major axis. CO was clearly detected by Thronson \& Bally (1987) in a large beam (100"). Our CO measurements refer to the center and do not include either of the major HII region complexes. This central part has also been mapped in $J=1-0{ }^{12} \mathrm{CO}$ at high resolution by Simon et al. (2003) using BIMA. Their map shows extended emission consistent with the equivalent $\mathrm{CO}$ size of about $1^{\prime}$ suggested by the single dish measurements. The values in Table 4 were calculated from the data in Tables 2 and 3 by assuming a $\mathrm{CO}$ source size of $60^{\prime \prime}$.

NGC 3077 (I0 pec, HII) is also a small member of the M 81 group. CO emission from this galaxy was discovered by Becker et al. (1989). Our measurements coincide with the peak in their map. High-resolution OVRO maps of the galaxy in the $J=1-0$ and $J=2-1 \mathrm{CO}$ transitions were published by Meier et al. (2001) and Walter et al. (2002) and show 
Table 3. Summary of literature ${ }^{12} \mathrm{CO}$ data on observed galaxies.

\begin{tabular}{|c|c|c|c|c|c|c|c|c|c|c|c|}
\hline$J$ & $\begin{array}{r}\text { Beam } \\
\left({ }^{\prime \prime}\right)\end{array}$ & $\begin{array}{c}I_{\mathrm{CO}} \\
\left(\mathrm{K} \mathrm{km} \mathrm{s}^{-1}\right)\end{array}$ & Ref. & $J$ & $\begin{array}{r}\text { Beam } \\
\left({ }^{\prime \prime}\right)\end{array}$ & $\begin{array}{c}I_{\mathrm{CO}} \\
\left(\mathrm{K} \mathrm{km} \mathrm{s}^{-1}\right)\end{array}$ & Ref. & $J$ & $\begin{array}{r}\text { Beam } \\
\left({ }^{\prime \prime}\right)\end{array}$ & $\begin{array}{c}I_{\mathrm{CO}} \\
\left(\mathrm{K} \mathrm{km} \mathrm{s}^{-1}\right)\end{array}$ & Ref. \\
\hline & & NGC 55 & & & & NGC 2976 & & & & NGC 4194 & \\
\hline \multirow[t]{2}{*}{$1-0$} & 43 & $3.40 \pm 0.40$ & 1 & $1-0$ & 100 & $1.20 \pm 0.20$ & 3 & $1-0$ & 100 & $1.55 \pm 0.19$ & 3 \\
\hline & & LGS 3 & & $1-0$ & 24 & $4.12 \pm 0.17$ & 5 & $1-0$ & 33 & $17.0 \pm 2.0$ & 11 \\
\hline \multirow[t]{2}{*}{$1-0$} & 45 & $0.76 \pm 0.15$ & 2 & $2-1$ & 11 & $1.98 \pm 0.30$ & 5 & $1-0$ & 24 & $29.3 \pm 0.26$ & 5 \\
\hline & & $2 \mathrm{Zw} 40$ & & & & Haro 2 & & $1-0$ & 22 & $49.0 \pm 3.0$ & 12 \\
\hline $1-0$ & 55 & $0.75 \pm 0.35$ & 3 & $1-0$ & 55 & $1.32 \pm 0.29$ & 1 & $1-0$ & 15 & $20.7 \pm 0.7$ & 13 \\
\hline $1-0$ & 45 & $0.62 \pm 0.15$ & 2 & $1-0$ & 55 & $1.13 \pm 0.26$ & 3 & $2-1$ & 22 & $66.0 \pm 4.0$ & 12 \\
\hline $1-0$ & 35 & $1.10 \pm 0.50$ & 4 & $1-0$ & 22 & $6.52 \pm 0.65$ & 6 & $2-1$ & 11 & $45.5 \pm 0.65$ & 5 \\
\hline $1-0$ & 24 & $0.23 \pm 0.05$ & 5 & $1-0$ & 22 & $3.8 \pm 0.3$ & 8 & $3-2$ & 14 & $17.7 \pm 0.4$ & 13 \\
\hline $1-0$ & 22 & $1.57 \pm 0.32$ & 6 & $2-1$ & 12 & $6.2 \pm 0.4$ & 8 & & & NGC 4214-S & \\
\hline $2-1$ & 12 & $0.88 \pm 0.16$ & 6 & $2-1$ & 12 & $6.21 \pm 0.36$ & 6 & $1-0$ & 55 & $0.65 \pm 0.10$ & 14 \\
\hline $2-1$ & 11 & $0.69 \pm 0.07$ & 5 & $3-2$ & 22 & $2.3 \pm 0.3$ & 7 & $1-0$ & 55 & $1.50 \pm 0.36$ & 15 \\
\hline \multirow[t]{2}{*}{$3-2$} & 22 & $<0.9$ & 7 & & & NGC 6052 & & & & NGC 5633 & \\
\hline & & NGC 2537 & & $1-0$ & 22 & $31.1 \pm 1.7$ & 6 & $1-0$ & 100 & $1.96 \pm 0.26$ & 3 \\
\hline $1-0$ & 24 & $0.91 \pm 0.13$ & 5 & $1-0$ & 17 & $23.3 \pm 1.0$ & 9 & & & & \\
\hline $1-0$ & 22 & $1.16 \pm 0.38$ & 6 & $1-0$ & 15 & $30.6 \pm 8.0$ & 10 & & & & \\
\hline $2-1$ & 12 & $1.12 \pm 0.17$ & 6 & $2-1$ & 12 & $23.8 \pm 0.7$ & 6 & & & & \\
\hline $2-1$ & 11 & $0.94 \pm 0.15$ & 5 & $3-2$ & 14 & $17.8 \pm 3.7$ & 10 & & & & \\
\hline $3-2$ & 22 & $<1.8$ & 7 & & & & & & & & \\
\hline
\end{tabular}

References: 1. Israel et al. (1995); 2. Tacconi \& Young (1987); 3. Thronson \& Bally (1987); 4. Gondhalekar et al. (1998); 5. Albrecht et al. (2004); 6. Sage et al. (1992); 7. Meier et al. (2001); 8. Barone et al. (2000); 9. Sofue et al. (1990); 10. Yao et al. (2003); 11. Aalto \& Hüttemeister (2000); 12. Casoli et al. (1992); 13. Devereux et al. (1994); 14. Taylor et al. (1998); 15. Thronson et al. (1988).

significant substructure. The ratios in Table 4 were derived from data by Becker et al. (1989), Meier et al. (2001) and Albrecht et al. (2004) for an equivalent CO source size of $25^{\prime \prime}$.

Haro 2 (Im pec, HII) is another BCG also known as Mkn 33. CO was detected by Thronson \& Bally (1987), Sage et al. (1992), Israel et al. (1995), Barone et al. (2000), and Meier et al. (2001) including measurement of the $J=2-1$ and $J=3-2{ }^{12} \mathrm{CO}$ transitions. An OVRO map of the $J=$ $1-0{ }^{12} \mathrm{CO}$ distribution was presented by Bravo-Alfaro et al. (2004) and shows that most of the emission originates in a source of size $15^{\prime \prime}-20^{\prime \prime}$ (cf. Fig. 2). Our $(2-1) /(1-0){ }^{12} \mathrm{CO}$ ratio is based on the mean of the $J=1-0$ CO fluxes in Table 3 . The implied (3-2)/(2-1) ratio appears to be dependent on the beamsize used. The mean ratio for the $21 / 22^{\prime \prime}$ beam is 0.63 ; whereas the the mean for the 12/14" beam is 1.08 . In Table 4 we list the average of these two.

NGC 3353 = Haro 3 = Mkn 35 (Irr, HII) is a BCG detected by Thronson \& Bally (1987), Tacconi \& Young (1987), Sage et al. (1992), and Meier et al. (2001). The available data only allow a reliable determination of the $(3-2) /(1-0)$ ratio, as the $J=2-1$ measurement by Sage et al. (1992) appears to be too low.

NGC 4194 (IBm pec, SB0 pec, HII) is also known as the BCG $1 \mathrm{Zw} 33$, Mkn 201 or the Medusa Merger. Measurements of the lower three ${ }^{12} \mathrm{CO}$ transitions have been published by Casoli et al. (1992), Devereux et al. (1994) and Aalto et al. (2001). An OVRO $J=1-0{ }^{12} \mathrm{CO}$ map was presented by Aalto \& Hüttemeister (2000). The equivalent CO size of $8^{\prime \prime}$ suggested by the single-dish measurements is consistent with the extent of the major $\mathrm{CO}$ concentration in their map. The data summarized in Table 3 are, unfortunately, not entirely consistent. In particular, we have decided to ignore the high $J=2-1{ }^{12} \mathrm{CO}$ value from Casoli et al. (1992), which is inconsistent with all other measurements. To obtain the $J=2-1 / J=$ 1-0 ratio, we have divided our $J=2-1$ value from Table 2 by the mean of the $22 / 24^{\prime \prime} J=1-0$ data in Table 3 . For the $J=3-2 / J=2-1$ ratio we have taken the mean of our own $\left(21^{\prime \prime}\right)$ values in Table $2(0.78)$ and the $11 / 14^{\prime \prime}$ values in that Table and in Table 3 (0.86). In view of the uncertainties, we have not attempted to correct the latter for finite source and beam sizes.

NGC 4214 (IAB(s)m, HII) was measured in ${ }^{12} \mathrm{CO}$ by Tacconi \& Young (1985), Thronson et al. (1988), Ohta et al. (1993), Becker et al. (1995), and Taylor et al. (1998). An OVRO map by Walter et al. (2000) shows three CO concentrations along the major axis separated by $40^{\prime \prime}$, each about $10-15^{\prime \prime}$ in size. Our measurements (cf. Fig 2) sample the southern maximum. The range of ratios in Table 4 refer to source sizes varying from great extent (high ratio) to essentially unresolved (low ratio). The (3-2)/(2-1) ratio cannot be determined accurately, but the upper limit is physically significant. 
Table 4. Integrated rotational line ratios in compact galaxies.

\begin{tabular}{lcccc}
\hline \hline Galaxy & $12+\lg [\mathrm{O}] /[\mathrm{H}]$ & $f_{60} / f_{100}$ & $\begin{array}{c}{ }^{12} \mathrm{CO}(2-1) /(1-0) \\
\left(R_{21}\right)\end{array}$ & $\begin{array}{c}\left.{ }^{12} \mathrm{CO}(3-2) /(2-1)\right) \\
\left(R_{32}\right)\end{array}$ \\
\hline IC 10-SE & 8.18 & 0.44 & $0.64 \pm 0.05$ & $0.98 \pm 0.14$ \\
LGS 3 & - & - & $0.4-1.8$ & $1.1-2.5$ \\
2 Zw 40 & 8.10 & 1.14 & $0.5:$ & $<1.5$ \\
NGC 2537 & - & 0.54 & $0.93 \pm 0.14$ & $<0.8$ \\
NGC 2976 & - & 0.35 & $0.96 \pm 0.07$ & $0.72 \pm 0.11$ \\
Haro 2 & 8.4 & 0.87 & $0.87 \pm 0.11$ & $0.86 \pm 0.23$ \\
NGC 4194 & - & 0.88 & $0.88 \pm 0.13$ & $0.82 \pm 0.11$ \\
NGC 4214-S & 8.27 & 0.57 & $0.3-1.3$ & $0.3-0.7$ \\
NGC 5633 & - & 0.34 & $0.5-2.7$ & $0.3-0.6$ \\
NGC 6052 & - & 0.70 & $0.86 \pm 0.17$ & $0.75 \pm 0.11$ \\
\hline NGC 3353 & 8.35 & 0.78 & & $-0.56 \pm 0.12-$ \\
\hline He 2-10 & $8.4-8.9$ & 0.91 & $0.97 \pm 0.16$ & $1.16 \pm 0.23$ \\
NGC 1569 & 8.19 & 0.90 & $1.15 \pm 0.10$ & $1.05 \pm 0.09$ \\
NGC 3077 & 9.02 & 0.59 & $0.83 \pm 0.16$ & $1.12 \pm 0.22$ \\
NGC 5253 & 8.15 & 1.05 & $1.43 \pm 0.29$ & $1.06 \pm 0.25$ \\
NGC 6822-HV & 8.21 & 0.55 & $1.28 \pm 0.30$ & $1.00 \pm 0.20$ \\
\hline
\end{tabular}

Table 5. Integrated isotopic line ratios in compact galaxies.

\begin{tabular}{lcccc}
\hline \hline Galaxy & \multicolumn{3}{c}{$\mathrm{CO} /{ }^{13} \mathrm{CO}$} & Ref. \\
& $J=1-0$ & $J=2-1$ & $J=3-2$ & \\
& $\left(r_{1}\right)$ & $\left(r_{2}\right)$ & $\left(r_{3}\right)$ & \\
\hline NGC 55 & $15 \pm 4$ & - & - & 1 \\
IC 10-SE & $9 \pm 1$ & $13 \pm 3$ & $12 \pm 2$ & 2 \\
He 2-10 & $20 \pm 4$ & $20 \pm 4$ & $16 \pm 5$ & 3 \\
NGC 1569 & $23 \pm 8$ & $36 \pm 9$ & - & 4 \\
NGC 4194 & $19 \pm 4$ & - & - & 5 \\
NGC 6822-HV & $23 \pm 7$ & - & $11 \pm 3$ & 6 \\
\hline
\end{tabular}

References: 1. Becker \& Freundling (1991); 2. Petitpas \& Wilson (1998); Bolatto et al. (2000); redetermined from archive data; 3. Baas et al. (1994); Kobulnicky et al. (1995); 4. Mühle (2003); 5. Aalto et al. (2001); 6. Israel et al. (2003), redetermined.

NGC 5253 (Impec, HII, Sbrst), also known as Haro 10, is a small but pronounced starburst member of the M 83 group. The $J=1-0$ observations in various beams (Wiklind \& Henkel 1989; Turner et al. 1997; Taylor et al. 1998) suggest an equivalent CO size of $35^{\prime \prime}$. This, together with the $J=2-1$ and $J=3-2$ obervations by Meier et al. (2001) implies the ratios given in Table 4.

NGC 5633 ((R)SA(rs)b) was detected in CO by Thronson $\&$ Bally (1987). No other CO measurements have appeared in the literature, so that we can only constrain possible ratios by the assumption of either pointlike or extended $\mathrm{CO}$ emission. Again, only the (3-2)/(2-1) ratio is sufficiently constrained to have physical meaning.

NGC 6052 is an otherwise unclassified BCG more commonly known as Mkn 297 with an optical size of about 20", possibly the result of a merger. Comparison of all available measurements suggests that Sage et al. (1992) overestimated the $J=1-0$ intensity; the ratio derived from $14^{\prime \prime}$ beam observations (assuming identical source structure in all CO transitions) is more plausible and given in Table 4. Our $J=3-2$ map (Fig. 3) shows the CO source to be resolved whereas the $J=$ 1-0 map by Sofue et al. (1990) only shows marginal resolution. Taken together, these results suggest a CO extent of about $15^{\prime \prime}$.

NGC 6822 is a Local Group galaxy similar to the LMC. The entries in Table 4 were taken from Israel et al. (2003) and refer to individual pointings on the $\mathrm{CO}$ cloud complex associated with the star-formation region Hubble V.

\section{Analysis and discussion}

\subsection{Physical condition of the gas}

The observed ${ }^{12} \mathrm{CO}$ and ${ }^{13} \mathrm{CO}$ transitions can be analyzed to provide constraints on the physical condition of the molecular gas in the compact galaxies concerned. To this purpose we have used the large-velocity gradient (LVG) radiative transfer models described by Jansen (1995) and Jansen et al. (1994). They provide model line intensities as a function of three input parameters: gas kinetic temperature $T_{\mathrm{k}}$, molecular hydrogen density $n\left(\mathrm{H}_{2}\right)$ and $\mathrm{CO}$ column density per unit velocity $(N(\mathrm{CO}) / \mathrm{d} V)$. Comparison of model to observed line ratios allows identification of the physical parameters best describing the actual conditions in the observed source. Beam-averaged properties are determined by comparing observed and model line intensities. Tables 4 and 5 show that in several galaxies the input parameters are not fully constrained by the observations. At least some constraint on gas conditions can be derived for NGC 2976, NGC 3077, Haro 2 (Mkn 33), NGC 4194 
Table 6. Physical parameters of model clouds.

\begin{tabular}{|c|c|c|c|c|c|c|c|c|c|c|c|c|c|c|}
\hline & $\begin{array}{l}\text { Kin. } \\
\text { Temp. }\end{array}$ & $\begin{array}{c}\text { Gas } \\
\text { Density }\end{array}$ & $\begin{array}{l}\text { Column } \\
\text { Density }\end{array}$ & $\begin{array}{l}\text { Contribution } \\
\text { to } J=2-1\end{array}$ & \multicolumn{5}{|c|}{$\begin{array}{c}\text { Observed } \\
\text { Ratios }\end{array}$} & \multicolumn{5}{|c|}{$\begin{array}{l}\text { Model } \\
\text { Ratios }\end{array}$} \\
\hline & $\begin{array}{l}T_{\mathrm{k}} \\
(\mathrm{K})\end{array}$ & $\begin{array}{l}n\left(\mathrm{H}_{2}\right) \\
\left(\mathrm{cm}^{-3}\right)\end{array}$ & $\begin{array}{c}N(\mathrm{CO}) / \mathrm{d} V \\
\left(\mathrm{~cm}^{-2} / \mathrm{km} \mathrm{s}^{-1}\right)\end{array}$ & Emission & $R_{21}$ & $R_{32}$ & $r_{1}$ & $r_{2}$ & $r_{3}$ & $R_{21}$ & $R_{32}$ & $r_{1}$ & $r_{2}$ & $r_{3}$ \\
\hline \multicolumn{15}{|c|}{ NGC 2537, NGC 2976, Haro 2, NGC 4194, NGC 6052} \\
\hline & 60 & 3000 & $0.6-1.0 \times 10^{17}$ & 1.00 & 0.91 & 0.80 & 15 & - & - & 0.93 & 0.78 & 16 & 9 & 14 \\
\hline \multicolumn{15}{|c|}{ IC 10-SE } \\
\hline 1 & 30 & $10^{4}-10^{5}$ & $1.0 \times 10^{17}$ & 0.15 & 0.64 & 0.98 & 9 & 13 & 12 & 0.76 & 0.93 & 11 & 11 & 13 \\
\hline 2 & 100 & 100 & $1.0 \times 10^{17}$ & 0.85 & & & & & & & & & & \\
\hline \multicolumn{15}{|c|}{ NGC 1569} \\
\hline 1 & 100 & $10^{5}$ & $1.0 \times 10^{17}$ & 0.25 & 1.15 & 1.05 & 23 & 36 & - & 1.23 & 0.99 & 23 & 20 & 18 \\
\hline 2 & 100 & 1000 & $0.3 \times 10^{17}$ & 0.75 & & & & & & & & & & \\
\hline \multicolumn{15}{|c|}{ He 2-10, NGC 3077} \\
\hline 1 & 60 & $10^{5}$ & $0.6 \times 10^{17}$ & 0.20 & 0.90 & 1.14 & 20 & 20 & 16 & 0.99 & 0.83 & 21 & 18 & 17 \\
\hline 2 & $100 \pm 50$ & $500-1000$ & $0.3 \times 10^{17}$ & 0.80 & & & & & & & & & & \\
\hline \multicolumn{15}{|c|}{ NGC 6822-HV, NGC 5253} \\
\hline 1 & 30 & $10^{5}$ & $0.6 \times 10^{17}$ & 0.75 & 1.35 & 1.03 & 23 & - & 11 & 1.45 & 0.99 & 22 & 14 & 11 \\
\hline 2 & $30-150$ & $10^{5}$ & $0.3 \times 10^{17}$ & 0.25 & & & & & & & & & & \\
\hline
\end{tabular}

(Mkn 201), NGC 5253, and NGC 6052 (Mkn 297). Sufficient information for a proper analysis is provided only by (the star-forming complexes in) IC 10-SE, NGC 1569, NGC 6822$\mathrm{HV}$ and $\mathrm{He} 2-10$.

\subsubsection{Limitations of single-component modelling}

First, we have attempted to fit the observed line ratios with a molecular gas at a single temperature, a single density and a single velocity gradient by searching a grid of model intensity ratios corresponding to temperatures $T_{\mathrm{k}}=$ $10-250 \mathrm{~K}$, densities $n\left(\mathrm{H}_{2}\right)=10^{2}-10^{5} \mathrm{~cm}^{-3}$, and gradients $N(\mathrm{CO}) / \mathrm{d} V=6 \times 10^{15}-3 \times 10^{18} \mathrm{~cm}^{-2} / \mathrm{km} \mathrm{s}^{-1}$ ) for values matching the observed intensity ratios. Among the galaxies listed in Table 4, five (NGC 2537, NGC 2976, Haro 2, NGC 4194 and NGC 6052) have almost identical line ratios with mean values $(2-1) /(1-0)=0.90$ and $(3-2) /(2-1)=$ 0.78 . These ratios are accurately reproduced (Table 6 ) by a warm and moderately dense molecular gas of temperature $T_{\text {kin }}=60(+40,-20) \mathrm{K}$, density $n\left(\mathrm{H}_{2}\right)=3000 \mathrm{~cm}^{-3}$, and gradient $N(\mathrm{CO}) / \mathrm{d} V=0.6-1.0 \times 10^{17} \mathrm{~cm}^{-2}\left(\mathrm{~km} \mathrm{~s}^{-1}\right)^{-1}$. The $J=$ $1-0{ }^{12} \mathrm{CO} /{ }^{13} \mathrm{CO}$ ratio in NGC 4194 (Table 5) agrees well with this. The intensities imply a filling factor $3.3 \times 10^{-3}$ and beamaveraged $\mathrm{CO}$ column-densities range from $\approx 1 \times 10^{16} \mathrm{~cm}^{-2}$ (NGC 2976, Haro 2) to $5 \times 10^{16} \mathrm{~cm}^{-2}$ (NGC 6052) to $1 \times$ $10^{17} \mathrm{~cm}^{-2}$ (NGC 4194). The relatively low $(2-1) /(1-0)$ ratios (mean: 0.56) for the Albrecht et al. (2004) galaxies (Sect. 3.2), and for $2 \mathrm{Zw} 40$ (Table 4), suggest the dominating presence of fairly low-density $\left(n\left(\mathrm{H}_{2}\right) \leq 800 \mathrm{~cm}^{-3}, N(\mathrm{CO}) / \mathrm{d} V=0.3-0.6 \times\right.$ $10^{17} \mathrm{~cm}^{-2}\left(\mathrm{~km} \mathrm{~s}^{-1}\right)^{-1} \mathrm{r}$ gas at undetermined temperatures.

The physical meaning of these results should be established by further observations, especially of the ${ }^{13} \mathrm{CO}$ isotope, but we suspect that additional information will only serve to rule out single-component fits after all. It is perhaps telling that no other galaxy in Table 4 is satisfactorily fitted by a single component. Moreover, even in cases where a single component appears to provide a good (LVG) fit to the observations, caution should be exercised in accepting the result as a physical reality, especially when the fit is based on limited data. This may be illustrated by the case of IC 10-SE. The relative intensities of the $J=1-0, J=2-1$ and $J=3-2{ }^{12} \mathrm{CO}$ lines together with the $J=1-0$ and $J=2-1{ }^{12} \mathrm{CO} /{ }^{13} \mathrm{CO}$ isotopic ratios are perfectly fitted by a single hot $\left(T_{\text {kin }}=100 \mathrm{~K}\right)$ and tenuous $\left(n\left(\mathrm{H}_{2}\right)=100 \mathrm{~cm}^{-3}\right)$ molecular gas component. Note that this result is based on a total of four line ratios. As many determinations in the literature are based on three or only two ratios, this is more than the number commonly used in such analyses. Yet, this apparently excellent fit completely fails to correctly predict the other two ratios measured for IC 10-SE. The observed modest $J=3-2{ }^{12} \mathrm{CO} /{ }^{13} \mathrm{CO}$ ratio of eleven is four times lower than predicted, and the modelled $J=4-3{ }^{12} \mathrm{CO}$ intensity falls short of the observed value by a factor of two or more.

Although this might suggest that single-component LVG analysis is physically irrelevant, this is not quite true. The kinetic temperatures and spatial densities implied by such fits often actually occur in the source. However, when they do, temperature and density generally do not refer to the same volume of gas, as will be clear from the following.

\subsubsection{Dual-component modelling}

For almost all of the sample galaxies, good fits based on two gas components could be obtained. Although the number of free parameters exceeds the number of independent measurements, this is a meaningful result because the actual combination of physically allowed parameters is sufficiently 
constrained. In order to reduce the number of free parameters, we have assumed identical CO isotopical abundances for both gas components and assign the specific value $\left[{ }^{12} \mathrm{CO}\right] /\left[{ }^{13} \mathrm{CO}\right]=40$. Different choices are possible, but reasonably small changes, for instance to ratios of 50 or 60 rarely lead to very different outcomes. We identified acceptable fits by searching a grid of model parameter combinations (covering the same range of temperatures, densities and gradients as described above) for matching model and observed line ratios, for various relative contributions of the two components.

We have rejected all solutions in which the denser gas component is also hotter than the more tenuous component, as we consider this physically unlikely on the large linear scales observed. From the remainder of solutions, we have selected characteristic examples and listed these in Table 6. The solutions are not unique, but delineate a range of values in particular parameter space regions. Variations in input parameters may compensate for one another, causing somewhat different input combination to yield identical line ratios, as indicated in the table entries.

The observed line ratios for the star-forming regions in all six galaxies listed in Table 5 require the presence of a rather dense component (typically $n_{\mathrm{H}_{2}}=10^{5} \mathrm{~cm}^{-3}$ ) but these components vary from fairly cool (IC 10-SE, NGC 6822-HV and probably NGC 5253) to warm (He2-10 and probably NGC 3077) to hot (NGC 1569). Column densities are well-established in the range $N(\mathrm{CO}) / \mathrm{d} V=0.6-1.0 \times$ $10^{17} \mathrm{~cm}^{-2} / \mathrm{km} \mathrm{s}^{-1}$. This dense component is associated with a second component that is generally less dense, but hotter (typically $100 \mathrm{~K})$. The actual density of this second component ranges from $n_{\mathrm{H}_{2}}=100 \mathrm{~cm}^{-3}$ (IC $10-\mathrm{SE}$ ) to $n_{\mathrm{H}_{2}} \approx 10^{3} \mathrm{~cm}^{-3}$ (NGC 1569, He 2-10 and probably NGC 3077). No signature of low-density gas is apparent in NGC 6822-HV and possibly NGC 5253. In these objects, the hot second component is still very dense (typically $10^{5} \mathrm{~cm}^{-3}$ ). Note that the notable physical difference between e.g. NGC 1569 and NGC 6822 is not immediately obvious as these objects have very similar ${ }^{12} \mathrm{CO}$ line ratios and identical $J=1-0{ }^{12} \mathrm{CO} /{ }^{13} \mathrm{CO}$ ratios. The important discriminators are the remaining ${ }^{12} \mathrm{CO} /{ }^{13} \mathrm{CO}$ ratios. A relatively low $J=3-2{ }^{12} \mathrm{CO} /{ }^{13} \mathrm{CO}$ ratio (NGC 6822) is incompatible with a significant presence of low-density gas at any temperature, whereas a high $J=2-1{ }^{12} \mathrm{CO} /{ }^{13} \mathrm{CO}$ (NGC 1569) requires the presence of very hot and very dense gas, or just low-density gas, or a combination of both. Even then, we find it difficult (as Table 6 shows) to reproduce the very high $J=2-1{ }^{12} \mathrm{CO} /{ }^{13} \mathrm{CO}$ apparently appropriate to NGC 1569 . This suggests that the observations are in error, or that our assumptions are incorrect. Neither possibility can be ruled out. NGC 1569 is one of the most extreme (post) starburst known (cf. Israel 1988) and its ISM appears to be subjected to intense processing (Lisenfeld et al. 2002). Thus, the less abundant ${ }^{13} \mathrm{CO}$ isotope may have selectively destroyed, so that its actual abundance is much less than assumed in our modelling. This would indeed lead to higher ${ }^{12} \mathrm{CO} /{ }^{13} \mathrm{CO}$ ratios. In the absence of further data we have chosen not to pursue this possibility, but it does suggest that (re)determination of the ${ }^{13} \mathrm{CO}$ intensities in NGC 1569 might be fruitful.

\subsection{Beam-averaged molecular gas properties}

The fraction of carbon locked up in the carbon monoxide molecule (i.e. the $N(\mathrm{CO}) / N_{\mathrm{C}}$ ratio) as given by the chemical models by Van Dishoeck \& Black (1988) is strongly dependent on the total carbon (and molecular hydrogen) column density. At column densities $N_{\mathrm{C}}>10^{18} \mathrm{~cm}^{-2}$ practically all gas-phase carbon is in CO, whereas at column densities $N_{\mathrm{C}}<10^{17} \mathrm{~cm}^{-2}$ essentially all carbon is in atomic form. Using these chemical models and the radiative transfer model parameters summarized in Table 6, we have estimated the beam-averaged carbon monoxide column densities as well as the beam-averaged column densities of all (atomic and molecular) carbon present in the gas-phase. Total carbon column densities $N_{\mathrm{C}}$ may be converted into total hydrogen column densities $N_{\mathrm{H}}$ as a function of the $[\mathrm{C}] /[\mathrm{H}]$ abundance and the fraction of carbon in the gas-phase. For galaxies with metallicities below solar, the $[\mathrm{C}] /[\mathrm{H}]$ abundance can be estimated from the $[\mathrm{C}] /[\mathrm{O}]$ versus $[\mathrm{O}] /[\mathrm{H}]$ diagrams given by Garnett et al. (1999) and the $[\mathrm{O}] /[\mathrm{H}]$ metallicities summarized in Table 4 . We furthermore assume a gas-phase carbon-depletion factor $\delta_{\mathrm{C}}=0.27$. By subtracting observed neutral hydrogen column densities $N(\mathrm{HI})$ from the total hydrogen column densities thus obtained, beamaveraged molecular hydrogen column densities are determined. Finally, these are used to obtain total molecular hydrogen masses and estimates of the CO-to- $\mathrm{H}_{2}$ conversion factor $X$. Table 7 gives the results for those galaxies where this procedure was meaningful. We find that at least in the low-metallicity starburst objects, overall CO column densities are low (which is reflected by the relatively low velocity-integrated intensities in Table 2). We also find that in these objects, only a small fraction of all carbon is in $\mathrm{CO}$ and that most carbon should be in neutral or ionized atomic form. As a consequence, molecular hydrogen densities are not proportionally lower. Low integrated $\mathrm{CO}$ intensities and relatively normal molecular hydrogen column densities together imply the high $X$-values given in Table 7 .

\subsection{Molecular gas in compact galaxies}

Although the database is admittedly still limited, we can make a few statements as to the general nature of molecular gas in compact and dwarf irregular galaxies. On the basis of $\mathrm{CO}$ spectroscopy we may distinguish different types of environment in compact galaxies, with properties summarized in Table 8. We have included results obtained in a similar way for a sample of high-metallicity starburst galaxy centers (NGC 253, IC 342, Maffei 2, M 83, and NGC 6946 - Israel et al. 1995; Israel \& Baas 2001, 2003) and for a quiescent centers such as occur in NGC 7331 (Israel \& Baas 1999) and the Milky Way (Bennett et al. 1994). The former are characterized by CO intensities slowly decreasing with increasing rotational transition, as well as ${ }^{12} \mathrm{CO} /{ }^{13} \mathrm{CO}$ ratios of about 10 . The latter are weaker $\mathrm{CO}$ emitters, more difficult to detect and less frequently included in surveys. They have CO intensities much more rapidly decreasing with rotational transition, and they have lower ${ }^{12} \mathrm{CO} /{ }^{13} \mathrm{CO}$ ratios around 6-7. Finally, we also included average ratios obtained for both quiescent and actively star-forming molecular clouds in the low-metallicity 
Table 7. Beam-averaged physical parameters.

\begin{tabular}{lcccccr}
\hline \hline Galaxy & \multicolumn{2}{c}{ Abundances } & \multicolumn{2}{c}{ Column density } & Mass & CO- $\mathrm{H}_{2}$ ratio \\
& $N_{\mathrm{C}} / N(\mathrm{CO})$ & $N_{\mathrm{H}} / N_{\mathrm{C}}$ & $\begin{array}{c}N(\mathrm{HI}) \\
\left(\mathrm{cm}^{-2}\right)\end{array}$ & $\begin{array}{c}N\left(\mathrm{H}_{2}\right) \\
\left(\mathrm{cm}^{-2}\right)\end{array}$ & $\begin{array}{c}M\left(\mathrm{H}_{2}\right) \\
\left(M_{\odot}\right)\end{array}$ & $\begin{array}{r}X \\
\left(\mathrm{~cm}^{-2} / \mathrm{K} \mathrm{km} \mathrm{s}^{-1}\right)\end{array}$ \\
\hline Haro 2 & $21 \pm 2$ & $4 \times 10^{4}$ & $1.6 \times 10^{21}$ & $5 \times 10^{21}$ & $7 \times 10^{7}$ & $9 \times 10^{20}$ \\
IC 10-SE & $5 \pm 1$ & $1 \times 10^{5}$ & $3.6 \times 10^{21}$ & $24 \times 10^{21}$ & $3 \times 10^{6}$ & $12 \times 10^{20}$ \\
NGC 1569 & $46 \pm 23$ & $1 \times 10^{5}$ & $3.7 \times 10^{21}$ & $11 \times 10^{21}$ & $1 \times 10^{7}$ & $72 \times 10^{20}$ \\
He 2-10 & $11 \pm 2$ & - & $1.9 \times 10^{21}$ & - & - & $<4 \times 10^{20}$ \\
NGC 6822-HV & $22 \pm 3$ & $6 \times 10^{4}$ & $1.6 \times 10^{21}$ & $10 \times 10^{21}$ & $5 \times 10^{5}$ & $60 \times 10^{20}$ \\
\hline
\end{tabular}

Note: HI column densities from Bravo-Alfaro et al. (2004: Haro 2), Madden et al. (1997: IC 10), Stil \& Israel (2002: NGC 1569), Kobulnicky et al. (1995: He 2-10), Israel et al. (2003: NGC 6822).

Table 8. Environment and molecular gas parameters.

\begin{tabular}{|c|c|c|c|c|c|c|c|c|c|}
\hline \multirow[t]{2}{*}{$\begin{array}{c}\text { Galaxy } \\
\end{array}$} & \multicolumn{3}{|c|}{${ }^{12} \mathrm{CO}$} & \multicolumn{3}{|c|}{${ }^{12} \mathrm{CO} /{ }^{13} \mathrm{CO}$} & \multirow[t]{2}{*}{ 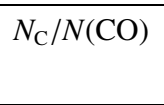 } & \multirow{2}{*}{$\begin{array}{c}\log N(\mathrm{CO}) \\
\left(\mathrm{cm}^{-2}\right)\end{array}$} & \multirow{2}{*}{$\begin{array}{c}\log N\left(\mathrm{H}_{2}\right) \\
\left(\mathrm{cm}^{-2}\right)\end{array}$} \\
\hline & $(2-1) /(1-0)$ & $(3-2) /(2-1)$ & $(3-2) /(1-0)$ & $(1-0)$ & $(2-1)$ & $(3-2)$ & & & \\
\hline \multicolumn{10}{|c|}{ Compact Galaxies } \\
\hline Type I (Starburst) & 1.1 & 1.1 & 1.2 & 20 & 23: & 13 & 21 & 16.6 & 22.2 \\
\hline Type II (Active) & 0.9 & 0.8 & 0.7 & 17: & - & - & 21 & 16.2 & 21.7 \\
\hline Type III (Quiet) & 0.5 & - & - & - & - & - & - & - & - \\
\hline \multicolumn{10}{|c|}{ Spiral Galaxy Centers } \\
\hline Starburst & 1.0 & 0.7 & 0.7 & 10 & 10 & 11 & 3 & 18.1 & 21.7 \\
\hline Quiet Nucleus & 0.5 & 0.6 & 0.3 & 7 & 6 & - & 6 & 18.0 & 21.6 \\
\hline \multicolumn{10}{|c|}{ LMC/SMC Clouds } \\
\hline Star-Forming & 1.1 & 0.9 & 1.0 & 12 & 8 & - & - & - & - \\
\hline Quiet & 0.7 & 0.5 & 0.4 & 10 & 14 & - & - & - & - \\
\hline
\end{tabular}

environments of the Large and the Small Magellanic Clouds (Bolatto et al. 2000, 2005).

Type I (starburst compact) has CO emission with $\int T_{\mathrm{mb}} \mathrm{d} V$ rising with increasing $J$-transition: both the $(2-1) / 1-0)$ and the $(3-2) /(2-1)$ ratios are close to or even exceeding unity. At the same time, the ${ }^{12} \mathrm{CO} /{ }^{13} \mathrm{CO}$ isotopical ratios are quite high, typically of the order of 20 . The CO results obtained towards NGC 1569, He 2-10, NGC 5253 and NGC 6822-HV are all in this category, as are those of the object UM 465 mentioned earlier. The molecular clouds sampled are dominated by intense star-formation activity. This may be representative of a large part of the galaxy (NGC 1569, He 2-10) or only of a specific location (NGC 6822-HV). The rotational line ratios imply high overall temperatures, and the isotopical ratios are characteristic of relatively low molecular gas beamaveraged column densities. This is clearly illustrated in Table 8. The line ratios of type I compact galaxies are very similar to those of spiral galaxy starburst centers and the low-metallicity starforming clouds in LMC and SMC, except for much higher ${ }^{12} \mathrm{CO} /{ }^{13} \mathrm{CO}$ ratios. This is characteristic of similar (high) temperatures but lower optical depths in the starburst dwarf galaxies. Hence, in the compact galaxies beam-averaged CO column densities are more than an order of magnitude lower than those in starburst galaxy centers and star-forming LMC/SMC clouds. In the compact galaxies most carbon is in atomic form. The beam-averaged molecular hydrogen columns are very similar to those of the starburst centers. The line ratios of IC 10-SE are different from any other set. They most resemble a mixture of active and cold LMC/SMC clouds.

Because integrated $\mathrm{CO}$ intensities are low and the $\mathrm{H}_{2}$ column densities implied by the analysis are not, $X$-factors in compact galaxies very significantly exceed those of starburst centers. We have calculated values $X=(12,72,60) \times$ $10^{20} \mathrm{~cm}^{-2}\left(\mathrm{~K} \mathrm{~km} \mathrm{~s}^{-1}\right)^{-1}$ for IC 10, NGC 1569 and NGC 6822 respectively. These values are in good agreement with conclusions reached earlier and independently for IC 10 by Madden et al. (1997) and for NGC 1569 by Lisenfeld et al. (2002). They also lend further support to the strong metallicity dependence of the $X$-factor proposed by Israel $(1997,2000)$.

Type II (active compact) has Haro 2 as a prototype. The line parameters are similar to those of type I objects, but the rotational line ratios are lower and less than unity. The difference is clearly seen in the ${ }^{12} \mathrm{CO}(3-2) /(1-0)$ ratio which is almost half that of the type I average. Most of the galaxies in the top panel of Table 4, including NGC 3353 (Haro 3) are of type II. They are actively forming stars, but the $\mathrm{CO}$ spectroscopy is not dominated by star-forming regions as in the type I objects. IC 10 and NGC 6822, now included in type I, would probably fall in this category if we had sampled the whole galaxy instead of only the most actively star-forming part. 
Even for Haro 2 the observational data are insufficient to allow a meaningful two-component analysis, so that the derived parameters are based only on an overly simplified single-component analysis. Nevertheless, as in type I objects, carbon monoxide appears to be only $\approx 5 \%$ of all gas-phase carbon. Carbon monoxide columns appear two orders of magnitude lower than those in starburst nuclei, but molecular hydrogen columns are virtually identical. For Haro 2 we find $X=9 \times 10^{20} \mathrm{~cm}^{-2}\left(\mathrm{~K} \mathrm{~km} \mathrm{~s}^{-1}\right)^{-1}$ practically identical to the value derived in a different manner by Barone et al. (2000). Although this is much lower than the average derived for the type I objects, it is very close to the value expected for this metallicity in the diagrams by Israel $(1997,2000)$. If we assume, just for the sake of argument, the same metallicity for all type II galaxies, we can perform an analysis similar to that of Haro 2 also for the other galaxies in this particular subsample. Not surprisingly, we obtain very similar $\mathrm{CO}, \mathrm{C}$ and $\mathrm{H}_{2}$ columns (with a mean $\log N\left(\mathrm{H}_{2}\right)=21.8$ ). For NGC 2537 and NGC 2976 we would find $X=10-30 \times 10^{20} \mathrm{~cm}^{-2} / \mathrm{K} \mathrm{km} \mathrm{s}^{-1}$, but for the much more distant galaxies NGC 4194 and NGC 6052 we would obtain $X$ values an order of magnitude lower, not substantially different from the Solar Neighborhood value $X=$ $2 \times 10^{20} \mathrm{~cm}^{-2} / \mathrm{K} \mathrm{km} \mathrm{s}^{-1}$. The fact that these are the most luminous galaxies in the sample (cf. Table 2 ) is probably more relevant to this result than the fact that they are the most distant galaxies.

In the type II objects, the ${ }^{12} \mathrm{CO}(3-2) /(1-0)$ ratio is practically constant (at about 0.67 ) over the full range of infrared colors $f_{60} / f_{100}$. This mean ${ }^{12} \mathrm{CO}(3-2) /(1-0)$ ratio is, in fact, identical to the mean of 0.66 found by Yao et et al. (2003) for a sample of 60 infrared-luminous galaxies (with individual values varying from from 0.22 to 1.72 ). Yao et al. also presented evidence suggesting $X$ to be lower than the Solar Neighbourhood value by an order of magnitude, making their sample more similar to our starburst centers than to our compact galaxies.

Type III (quiet compact) consists of those galaxies where low $(2-1) /(1-0)$ line ratios $\sim 0.5$ suggest that on the whole little is going on. Their molecular gas is not very dense, and may also not be very warm. $2 \mathrm{Zw} 40$ is a member of this category, weak in $J=1-0$, barely detected in $J=2-1$, and not in $J=3-2$. This class also apears to include NGC 2730 , NGC 3659, NGC 4532, NGC 6570, NGC 7732, and IC 3521 from the sample observed by Albrecht et al. (2004). It would be of interest to determine further line ratios in order to constrain the physical conditions of the molecular gas, but the weakness of emission in all but the $J=1-0$ transition makes this a difficult task.

\section{Conclusions}

1. We have observed ${ }^{12} \mathrm{CO}$ line emission in the $J=2-1$ and $J=3-2$ transitions from 11 compact (dwarf) galaxies. In four cases, limited maps were made.

2. By combining our data with those from the literature, we eliminated beam-dilution effects and established accurate line ratios for the first three ${ }^{12} \mathrm{CO}$ rotational transitions in a sample of a dozen objects, and limited the range of possible values in another four. For six objects, ${ }^{12} \mathrm{CO} /{ }^{13} \mathrm{CO}$ isotopical ratios were culled from the literature.

3. Radiative-transfer (LVG) modelling shows that in most of the observed galaxies warm molecular gas occurs with temperatures typically $T_{\mathrm{k}}=50-150 \mathrm{~K}$ as well as dense gas with $n\left(\mathrm{H}_{2}\right) \geq 3000 \mathrm{~cm}^{-3}$. Models using two distinct molecular gas components produce results different from and better than those obtained by modelling only a single component.

4. Chemical modelling implies that in the observed galaxies only a small fraction (typically $5 \%$ ) of all gas-phase carbon is in the form of $\mathrm{CO}$, the remainder being in the form of neutral carbon ([CI]) and especially ionized carbon ([CII]). As a consequence, $\mathrm{CO}$ column densities are quite low and of the order of $10^{16} \mathrm{~cm}^{-2}$.

5. Molecular hydrogen column densities are high, of the order of $10^{22} \mathrm{~cm}^{-2}$, and confirm the large CO-to- $\mathrm{H}_{2}$ conversion factors, in the range $X=10^{21}-10^{22} \mathrm{~cm}^{-2} / \mathrm{K} \mathrm{km} \mathrm{s}^{-1}$, found earlier for low-metallicity environments by different methods.

6. The CO spectroscopy of compact galaxies may be classified into three different types. Type I is characterized by high rotational and isotopic ratios, and reflects hot and dense molecular clouds dominated by starforming regions. Type II has lower ratios, in particular the ${ }^{12} \mathrm{CO}(3-2) /(1-0)$ ratio is much lower than in type I, and identical to the mean found for infrared-luminous galaxies in general. Type III has a low ${ }^{12} \mathrm{CO}(2-1) /(1-0)$ ratio indicative of not very dense and possibly relatively cool molecular gas, and appears to represent quiescent compact galaxies.

Acknowledgements. We thank JACH personnel, in particular Fred Baas ( $\dagger$ ), Remo Tilanus and Göran Sandell for their help in obtaining the observations discussed in this paper, and the referee, Ute Lisenfeld, for critical remarks leading to improvements in the paper.

\section{References}

Aalto, S., \& Hüttemeister, S. 2000, A\&A, 362, 42

Aalto, S., Hüttemeister, S., \& Polatidis, A. G. 2001, A\&A, 372, L29

Albrecht, M., Chini, R., Krügel, E., Müller, S. A. H., \& Lemke, R. 2004, A\&A, 414, 141

Arnault, P., Caspoli, F., Combes, F., \& Kunth, D. 1988, A\&A, 205, 41

Baas, F., Israel, F. P., \& Koornneef, J. 1994, A\&A, 284, 403

Barone, L. T., Heithausen, A., Hüttemeister, S., Fritz, T., \& Klein, U. 2000, MNRAS, 317, 649

Beck, S. C., Turner, J. L., Langland-Shula, L. E., et al. 2002, AJ, 124, 2516

Becker, R., \& Freundling, W. 1991, A\&A, 251, 454

Becker, R., Schilke, P., \& Henkel, C. 1989, A\&A, 211, L19

Becker, R., Henkel, C., Bomans, C., \& Wilson, R. T. L. 1995, 295, 302

Bennett, C. L., Kogut, A., Hinshaw, G., et al. 1994, ApJ, 436, 423

Bolatto, A. D., Jackson, J. M., Wilson, C. D., \& Moriarty-Schieven, G. 2000, AJ, 532, 909

Bolatto, A. D., Israel, F. P., \& Martin, C. 2005, ApJ, in preparation

Boselli, A., Lequeux, J., \& Gavazzi, G. 2002, A\&A, 384, 33

Braine, J., Duc, P.-A., Lisenfeld, U., et al. 2001, A\&A, 378, 51 
Bravo-Alfaro, H., Brinks, E., Baker, A. J., Walter, F., \& Kunth, D. 2004, AJ, 127, 264

Brinks, E., \& Klein, U. 1988, MNRAS, 231, 63p

Casoli, F., Dupraz, C., \& Combes, F. D. 1992, A\&A, 264, 55

Chyzy, K. T., Knapik, J., Bomans, D. J., et al. 2003, A\&A, 405, 513

Dettmar, R.-J., \& Heithausen, A. 1989, ApJ, 344, L61

Devereux, N., Taniguchi, Y., Sanders, D. B., Nakai, N., \& Young, J. S. 1994, AJ, 107, 2006

Elmegreen, B. G., Elmegreen, D. M., \& Morris 1980, ApJ, 240, 455

Garnett, D. R., Shields, G. A., Peimbert, M., et al. 1999, ApJ, 513, 168

Gil de Paz, A., Silich, S. A., Madore, B. F., et al. 2002, ApJ, 573, L101

Gondhalekar, P. M., Johansson, L. E. B., Brosch, N., Glass, I. S., \& Brinks, E. 1998, A\&A, 335, 152

Gordon, M. A., Heidmann, J., \& Epstein, E. E. 1982, PASP, 94, 115

Greve, A., Becker, R., Johansson, L. E. B., \& McKeith, C. D. 1996, A\&A, 312, 391

Hodge, P. A., \& Lee, M. G. 1990, PASP, 102, 26

Hunter, D. A., \& Sage, L. 1993, PASP, 105, 374

Israel, F. P. 1988, A\&A, 194, 24

Israel, F. P. 1997, A\&A, 328, 471

Israel, F. P. 2000, in Molecular Hydrogen in Space, ed. F. Combes, \& G. Pineau des Forêts (Cambridge Univ. Press), 293

Israel, F. P., \& Burton, W. B. 1986, A\&A, 168, 369

Israel, F. P., White, G. J., \& Baas 1995, A\&A, 302, 343

Israel, F. P., \& Baas, F. 1999, A\&A, 351, 10

Israel, F. P., \& Baas, F. 2001, A\&A, 371, 433

Israel, F. P., \& Baas, F. 2003, A\&A, 404, 495

Israel, F. P., Tacconi, L. J., \& Baas, F. 1995, A\&A, 295, 599

Israel, F. P., Baas, F., Rudy, R. J., Skillman, E. D., \& Woodward, C. E. 2003, A\&A, 397, 87

Jansen, D. J. 1995, Ph.D. Thesis University of Leiden (NL)

Jansen, D. J., van Dishoeck, E. F., \& Black, J. H. 1994, A\&A, 282, 605

Kobulnicky, H. A., Dickey, J. M., Sargent, A. I., Hogg, D. E., \& Conti, P. S. 1995, AJ, 110, 116

Kobulnicky, H. A., \& Skillman, E. D. 1995, ApJ, 454, L121

Leroy, A., Bolatto, A. D., Simon, J. D., \& Blitz, L. 2005, ApJ, in press

Lisenfeld, U., Israel, F. P., Stil, J. M., \& Sievers, A. 2002, A\&A, 382, 860
Lonsdale, C. J., et al. 1989, Cataloged Galaxies and Quasars Observed in the IRAS Survey, Version 2, JPL D-1932

Madden, S. C., Poglitsch, A., Geis, N., Stacey, G. J., \& Townes, C. H. 1997, ApJ, 483, 200

Meier, D. S., Turner, J. L., Crosthwaite, L. P., \& Beck, S. C. 2001, AJ, 121,740

Meier, D. S., Turner, J. L., \& Beck, S. C. 2001, AJ, 122, 1770

Melisse, J. P. M., \& Israel, F. P. 1994, A\&AS, 103, 391

Mühle, S. 2003, Ph.D. Thesis, Univ. Bonn (FRG)

Ohta, K., Tomita, A., Saito, M., Sasaki, M., \& Nakai, N. 1993, PASJ, 45, L21

Petitpas, G. R., \& Wilson, C. D. 1998, ApJ, 496, 226

Sage, L. J., Salzer, J. J., Loose, H.-H., \& Henkel, C. 1992, A\&A, 265, 19

Simon, J. D., Bolatto, A. D., Leroy, A., \& Blitz, L. 2003, ApJ, 596, 957

Stil, J. M., \& Israel, F. P. 2002, A\&A, 382, 860

Tacconi, L. J., \& Young, J. S. 1985, ApJ, 290, 602

Tacconi, L. J., \& Young, J. S. 1987, ApJ, 322, 681

Taylor, C. L., Kobulnicky, H. A., \& Skillman, E. D. 1998, AJ, 116, 2746

Taylor, C. L., Hüttemeister, S., Klein, U., \& Greve, A. 1999, A\&A, 349,424

Thronson, H. A., \& Bally, J. 1987, ApJ, 319, L63

Thronson, H. A., Hunter, D. A., Telesco, C. M., Greenhouse, M., \& Harper, D. A. 1988, ApJ, 334, 605

Thronson, H. A., Hunter, D. A., Casey, S., \& Harper, D. A. 1990, ApJ, 355,94

Turner, J. L., Beck, S. C., \& Hurt, R. L. 1997, ApJ, 474, L11

Van Dishoeck, E. F., \& Black, J. H. 1988, ApJ, 334, 771

Walter, F., Taylor, C. L., Hüttemeister, S., Scoville, N., \& McIntyre, V. 2001, AJ, 121, 727

Walter, F., Weiss, A., Martin, C., \& Scoville, N. 2002, AJ, 123, 225

Wiklind, T., \& Henkel, C. 1989, A\&A, 225, 1

Yang, H., \& Skillman, E. D. 1993, AJ, 106, 1448

Yao, L., Seaquist, E. R., Kuno, N., \& Dunne, L. 2003, ApJ, 588, 771

Young, L. M., \& Lo, K. Y. 1997, ApJ, 490, 710 